\title{
Occupational allergy in an entomological research centre. I Clinical aspects of reactions to the sheep blowfly Lucilia cuprina
}

\author{
G L KAUFMAN, ${ }^{2}$ B H GANDEVIA, ${ }^{2}$ T E BELLAS, ${ }^{3}$ E R TOVEY, 4 B A BALDO 4 \\ From the Allergy Clinic, 'Royal North Shore Hospital of Sydney, St Leonards, NSW, Department of \\ Respiratory Medicine, ${ }^{2}$ Prince of Wales Hospital, Randwick, NSW, Commonwealth Scientific and Industrial \\ Research Organization Division of Entomology, ${ }^{3}$ Acton, ACT, and Kolling Institute of Medical Research, ${ }^{4}$ Royal \\ North Shore Hospital of Sydney, St Leonards, NSW, Australia
}

ABSTRACT Twenty eight per cent (15) of 53 workers engaged in a sheep blowfly breeding programme designed to control genetically the pest Lucilia cuprina experienced allergic manifestations resulting from contact with this insect. The most common symptoms were rhinitis, affected eyes, rashes, and lower respiratory symptoms, usually, but not always, immediate in type. A personal history of noninsect related asthma, allergic rhinitis, or eczema, or a combination of these was more common in the fly allergic group but some workers experienced allergic symptoms only when exposed to the adult sheep blowfly. Raised levels of serum IgE antibodies specific for adult and larval allergens were found in approximately $70 \%$ of symptomatic workers, whereas only $30 \%$ and $7 \%$ of two groups of asymptomatic workers were found to have these antibodies. It is concluded that the sheep blowfly is an important source of airborne allergens and can prove a considerable occupational health hazard. Measures designed to reduce worker contact with blowflies and their emanations considerably reduced the incidence of allergic symptoms in the exposed workers.

The true flies, members of the insect order Diptera, are widespread and common in our environment and many species impinge on our health and wellbeing. Some, such as mosquitoes, are obligate blood feeders and are not only a nuisance but can also act as vectors of disease. Others, such as the buffalo fly, attack livestock whereas others, such as fruit flies, infest food crops.

Species from several families of the Diptera have been implicated in inhalant allergies including the house flies (Muscidae), ${ }^{12}$ non-biting midges (Chironomidae), ${ }^{3}$ mushroom flies (Phoridae), ${ }^{4}$ moth flies (Psychodidae), ${ }^{5}$ blowflies (Calliphoridae), ${ }^{6}$ vinegar flies (Drosophilidae), ${ }^{7}$ mosquitoes (Culicidae), ${ }^{8}$ and black fungus gnats (Sciaridae). ${ }^{9}$ Some of the exposures have been to natural populations but most examples have been found among those exposed in the course of their work.

The larvae of calliphorid flies are flesh feeders; most feed on carrion but some species attack living animals. During the large scale control programme against the New World screwworm, ground staff and air crew

Accepted 25 July 1988 developed allergic conditions, including asthma, through exposure to the flies while packing and distributing boxes containing sterile adults. ${ }^{10-12}$

The Australian sheep blowfly, Lucilia cuprina, which is reared for genetic studies and as part of a biological control programme, has also caused occupational allergies through exposures of laboratory workers to larvae and to adult flies. ${ }^{13}$ After this recent demonstration of inhalant allergy to $L$ cuprina, workers engaged in an institute breeding blowflies were studied for sensitivity to this species. The study was based on a series of administered questionnaires which were epidemiologically based but added clinical queries when positive answers were elicited. Most previous reports of insect allergy have recorded diagnosis rather than symptoms; we report the latter in some detail to illustrate their diversity and because the pattern of symptoms may perhaps be related to mechanisms or pathogenesis or both.

\section{Materials and methods}

The subjects of this study were employees of a large entomological research institute in the ACT Australia, 
where for 50 years the sheep blowfly has been the subject of continuing research. Each subject had either current or past occupational exposure to the sheep blowfly and, in all cases, this included direct contact with the insect either in the rearing programme, field work, or in the laboratory. The period of work exposure to the insect ranged from at least three months to 30 years and varied from half an hour to 10 hours a day for up to seven days a week. The intensity of exposure often varied for each subject during the contact period but had generally increased in the preceding 20 years as the breeding programme was upgraded.

The study, undertaken in March 1985, consisted of a series of questionnaires administered by BG and GK, both of whom are experienced in surveys of occupational allergies. Fifteen millilitres of blood was collected from each subject for specific IgE antibody determinations. A preliminary survey provided information on each workers' duties and the insects with which they had worked.

The first part of the questionnaire dealt with smoking history, respiratory complaints, and allergic manifestations unrelated to known insect exposure, including a history of asthma, allergic rhinitis, and eczema. In the second part of the questionnaire subjects were asked whether they considered that occupational exposure to insects in their workplace had produced symptoms. The insects implicated were listed and the subjects describing sensitivity to sheep blowfly were identified. A special effort was made to identify any other source of symptoms that might masquerade as sensitivity to insects, such as insect foods. Where doubt existed as to the cause of symptoms, the subject was placed in the non-allergic group. Clinical details relating to the manifestations of insect allergy and a detailed work history with special reference to contact with sheep blowfly were obtained. Measures that had been taken to prevent or minimise symptoms were explored and their effectiveness evaluated.

RADIOALLERGOSORBENT TEST (RAST)

This was carried out using nitrocellulose $\operatorname{discs}^{14}$ as previously described. ${ }^{13}$

\section{Results}

CLASSIFICATION OF SUBJECTS ON THE BASIS OF CASE HISTORIES, ASSESSMENT OF

QUESTIONNAIRE, AND PHYSICAL EXAMINATION

Fifty four subjects with an occupational exposure to $L$ cuprina were studied. This number represented more than $95 \%$ of the exposed workers employed in the entomology research institute at the time of the study. Omission from the study was only through absence
Table 1 Clinical manifestations in the 15 subjects symptomatic to the sheep blowfly L cuprina

\begin{tabular}{lll}
\hline Syndrome & No of subjects & $\begin{array}{l}\% \text { Of exposed } \\
\text { workforce } \\
(n=54)\end{array}$ \\
\hline Upper respiratory tract & 13 & 24 \\
Eyes & 11 & 20 \\
Skin & 9 & 17 \\
Asthma/chest tightness & 6 & 11 \\
\hline
\end{tabular}

during the survey. A positive answer to epidemiological type questions relating to insect allergy led to further questions relating to clinical features of the allergy.

Fifteen subjects $(28 \%)$ reported allergic symptoms which were ascribed to $L$ cuprina. Nine $(17 \%)$ of the remaining 39 subjects reported symptoms on exposure to an insect other than $L$ cuprina. Details of studies with other insect species will be published separately. Thirty subjects $(56 \%)$ were not allergic to any insect.

Thirty eight subjects who had worked with $L$ cuprina still had current contact with the insect or had ceased working with it within the previous 12 months, including 14 of the 15 subjects who were allergic to the insect. The remaining allergic individual had ceased working with the insect two and a half years earlier. The other 15 subjects who were not allergic to $L$ cuprina had suspended occupational exposure to the blowfly within a perod ranging from 14 months to 19 years.

Each of the subjects allergic to sheep blowfly experienced one or more symptom complexes. Asthma and chest tightness could not be distinguished by history alone and were therefore grouped together. The most common complaint related to upper respiratory symptoms (rhinitis). This was followed in order by affected eyes, rashes, and asthma/chest tightness (table 1). The last was experienced by six workers.

\section{DURATION OF EXPOSURE}

The duration of exposure to $L$ cuprina was about the same for the blowfly allergic group (mean 8 years, range 1.5 to 27 ), for the non-allergic group who had had continuing exposure within the previous 12 months (mean 6 years, range 0.5 to 30 ), and the nonallergic individuals whose exposure had ceased more than 12 months previously (mean 6 years, range 1 to 16).

\section{CLINICAL FEATURES OF SUBJECTS}

Asthma/chest tightness

Six workers reported asthma or chest tightness or both, with occupational exposure to L cuprina and all six experienced work related rhinitis and conjunc- 
tivitis, while five also described rashes. Two had a history of asthma whereas the other four developed asthma exclusively on insect exposure. The individuals had worked with $L$ cuprina for periods ranging from two to 16 years and the period before symptoms first appeared ranged from six months to 11 years from the time of first work contact. All six subjects had daily work exposure to L cuprina, usually five to seven hours duration, and only one subject discontinued work with this insect because of symptoms. Adoption of occupational hygiene measures either eliminated the asthma and chest tightness or produced symptomatic improvement. Symptoms of asthma or chest tightness, or both, began within 45 minutes of starting work and in four workers sometimes persisted into the evening. One subject experienced spontaneous resolution of all symptoms after six months of allergic manifestations. Another subject described bouts of "pleuritic" type pain, as well as asthma, lasting several days after prolonged heavy exposure.

\section{Upper respiratory symptoms}

Thirteen workers experienced rhinitis specifically related to exposure to L cuprina. This consisted of variable combinations of nasal obstruction, rhinorrhoea, sneezing, itch in the nose or pharynx, or both, nasal or pharyngeal dryness, or both, and soreness and nasal crusting. Nine of these also had a history of nonoccupational rhinitis. Onset of symptoms occurred within 30 minutes of work exposure in 11 subjects but in two subjects there was a delay of two to four hours. Symptoms first appeared from six months to 28 years from the date of first exposure. The duration and intensity of exposure varied from a few hours a week to eight hours a day for six days a week. In some individuals the onset of symptoms could be related to a change in work procedures, resulting in much heavier exposure to flies. Symptoms were sufficiently mild or ameliorated by preventive measures such that only three workers had to abandon duties temporarily. Measures used to reduce inhalant exposure by 12 subjects included introduction of a local air extractor system, the use of a respirator, wet cleaning of cages, benches, etc, and the wearing of protective apparel including a mask. These resulted in the elimination, or pronounced reduction, of symptoms in nine workers.

\section{Eyes}

Eleven subjects experienced eye symptoms variably characterised by itch, pain, lachrymation, erythema, and oedema. In some cases these could be related to hand to eye contact, although seven insisted that symptoms occurred in the absence of such direct contact. There was no clear distinction in the pattern of symptoms between these two groups. Symptoms arose either immediately after exposure or were delayed for several hours. Similarly, symptoms either subsided within 30 minutes or persisted for several days. Onset of symptoms occurred from two to 16 years after first exposure to the fly. Daily work exposure was the same as set out above under the upper respiratory symptoms group. Eye symptoms were sufficiently mild for workers to persevere with their job.

\section{Skin}

Nine workers experienced dermatological reactions. In six of the subjects this took the form of immediate, localised pruritis or urticaria, or both, which resolved spontaneously within one hour. In the remaining three workers reactions developed from one to eight hours after contact and persisted for from several hours to days. In two of these the skin reaction took the form of generalised urticaria. Symptoms first developed from within weeks to 11 years after first exposure. Preventive measures such as the wearing of coveralls and masks and application of a barrier cream either eliminated or improved symptoms in six workers. After introduction of these measures, two workers experienced symptoms only on heavy exposure.

\section{Multiple symptoms}

Twelve of the 15 subjects diagnosed as allergic to the sheep blowfly had symptoms affecting more than one system. Two of the remaining three subjects had upper respiratory tract symptoms only and the other had only skin symptoms. Although at the time of the study all six subjects who had asthma/chest tightness also had at least one other symptom complex, analysis of the intervals before the onset of symptoms showed that asthma/chest tightness was not necessarily the last manifestation, although upper respiratory symptoms tended either to precede it or arise concurrently.

\section{SUBJECTS WITH NON-INSECT RELATED ALLERGY}

Atopy, as defined by a personal history of non-insect related allergic rhinitis, asthma, or eczema, or a combination of these, was reported by 10 of the 15 subjects $(67 \%)$ with symptoms related to sheep blowfly exposure. Twenty three of the 38 subjects $(59 \%)$ who were not sensitive to the blowfly gave a history of non-insect related allergy. When the nine subjects who gave a history of allergic symptoms on exposure to an insect other than blowfly were excluded from the control group, the group was reduced to 30, 15 of whom had a history of allergy to sources other than insects.

\section{SMOKING}

The prevalence of lifetime non-smokers was equal among workers allergic to sheep blowfly $(47 \%)$ and both the 39 subjects exposed to, but not allergic to, this 
Table 2 Results of radioallergosorbant tests (RASTS) for the presence of serum IgE antibodies specific for blowfly (L cuprina) allergens*

\begin{tabular}{|c|c|c|c|c|c|}
\hline \multirow[b]{2}{*}{ Group } & \multicolumn{5}{|l|}{$R A S T$} \\
\hline & No $(n=54)$ & Negative $†$ & Equivocal & Positive $\ddagger$ & Not done \\
\hline Symptomatic to $L$ cuprina & 15 & $4(27 \%)$ & $\mathbf{0}$ & $10(67 \%)$ & $1(7 \%)$ \\
\hline $\begin{array}{l}\text { Asymptomatic. } \\
\text { Contact with } L \text { cuprina within } 12 \text { months }\end{array}$ & 24 & $14(61 \%)$ & $1(4 \%)$ & $8(33 \%)$ & $1(4 \%)$ \\
\hline $\begin{array}{l}\text { Asymptomatic. } \\
\text { No contact with } L \text { cuprina over } 12 \text { months }\end{array}$ & 15 & $12(80 \%)$ & $2(13 \%)$ & $1(7 \%)$ & 0 \\
\hline
\end{tabular}

*Extract of whole adult fly used in the RAST.

$\dagger<2.5$ times uptake of ${ }^{125} \mathrm{I}$-antigen-IgE when used with cord serum.

$\ddagger>3.5$ times uptake of ${ }^{125}$ I-antigen-IgE when used with cord serum.

insect as well as the subgroup of 30 who reported no allergic symptoms to any insect. Similarly, smokers were equally represented in the three groups (33-37\%) whether this was defined as current smokers only or taken to include ex-smokers of less than six years. Five of the six with lower respiratory tract symptoms had never smoked.

\section{RASTS FOR IgE ANTIBODIES TO L CUPRINA ALLERGENS}

Serum was obtained from 14 of the 15 subjects allergic to sheep blowfly and from 38 of the remaining subjects who worked with the blowfly and who were not allergic to the fly. Results of RAST studies on these sera are summarised in table 2 . The RAST results obtained with solid phases prepared from extracts of sheep blowfly larvae generally mirrored the findings obtained when blowfly adult extracts were used. Ten out of the $15(67 \%)$ subjects symptomatic to the blowfly showed a positive RAST to adult $L$ cuprina allergens. Thirty three per cent of the 24 asymptomatic workers who were either currently working with this insect or had ceased within the previous 12 months had raised RAST scores. Only one of 15 asymptomatic workers, whose last contact with this insect had been over 12 months previously, had a raised RAST score.

\section{Discussion}

We have previously shown that inhaled materials derived from the sheep blowfly $L$ cuprina may produce allergic symptoms. ${ }^{13}$ In the present study the importance of the sheep blowfly as an occupational source of inhalant allergy has been defined. Twenty eight per cent of the exposed workforce developed allergic symptoms to this insect, a level similar to that reported for other insects after occupational exposure..$^{15-17}$

We found a high incidence of raised serum IgE antibodies specific for blowfly allergens. Two thirds of the subjects symptomatic to the blowfly _showed a positive RAST to $L$ cuprina allergens whereas asymptomatic workers who had had contact with sheep blowfly within 12 months showed a highepor incidence of insect specific IgE antibodies than workers who had not been in contact with this insect for 12 months or more. This suggests that serologica ${ }^{5}$ evidence of sensitisation may wane after prolongedP freedom from exposure. In the case of the common house fly Musca domestica, it has been suggested thaE "IgE antibody levels may be sufficiently responsive to allergen exposure for serial measurements to be usefubo as a biologic monitor of exposure after changes in work practice."' In relation to this conclusion however, it should be remembered that many inseck species show pronounced allergenic cross reaction ${ }^{18} 12$ and continued sensitisation may occur from contact with insects in the occupational setting, in the wildळ and in the home as well as in leisure pursuits. ${ }^{1-610-13202} \stackrel{2}{\vec{P}}$

The lack of a complete correlation between allergie symptoms and the presence of blowfly specific serumb IgE antibodies suggests either some deficiency in the RAST used (such as lack of sensitivity or specificity or that in some patients IgE antibodies did not participate in the mechanism of mediator release. One subject, whose severe allergic symptoms resolved spontaneously, showed a low serum RAST score. raising the possibility that immunological tolerance may have developed. In keeping with the findings of Spieksma et al pertaining to Drosophila melano gaster, ${ }^{17}$ we found no clear relation between the duration of occupational contact with the sheepblowfly and the risk of developing allergic symptoms nor with the localisation of the symptoms to nose eyes, skin, or bronchi. Heavy or long exposure, of both, was required to elicit symptoms in somes individuals while others reacted to relatively minor of short exposures, or both. In addition, we found nq clear association between severity of symptoms to the blowfly and RAST score.

Two patterns of symptom response could be recog nised. In one case symptoms appeared within half and hour of exposure; in the other case symptoms were delayed by from one to several hours. This differences may reflect a diversity of immunological mechanisms 
as there was no correlation between the level of specific IgE antibodies and either mode of presentation. Nor did the level of serum antibody relate to the type or number of allergic manifestations. Clearly other factors are operative in determining the type of clinical response elicited by insect allergens.

In the subjects who had multiple symptoms there was no consistent pattern to the order of symptom presentation except that upper respiratory symptoms either preceded or arose concurrently with lower respiratory manifestations. Although all subjects who had asthma or chest tightness also experienced other allergic symptoms, lower airway involvement was not always the last to present and it cannot be said that the presence of other allergic symptoms is a forerunner of lower respiratory involvement.

Subjects with asthma or chest tightness occasionally continued to get these symptoms on exercise or exposure to cold air for some months after cessation of insect contact, presumably reflecting persisting bronchial hyperreactivity.

Previously, it has been observed that atopic subjects are more likely to develop inhalant allergy from exposure to insects. ${ }^{1522}$ In our study, although clinical evidence of atopy was more frequent in those developing allergic manifestations to the fly, the difference did not reach statistical significance. In this inland city surrounded by grassland seasonal rhinitis is common and may partly account for the lack of a definite difference. Pre-employment or pre-placement screening for atopy cannot be justified except perhaps as a basis for advising a particular applicant to take special care. Screening for atopy is usually based on skin tests which were not done in the initial survey for logistic reasons but which will be included as part of a follow up study.

For most subjects, symptoms were not troublesome enough to interfere seriously with work duties. A minority, however, found it necessary to change jobs and most modified their work routine. Measures incorporating a number of general principles of occupational hygiene, and based on the findings of a survey conducted by the United States National Institute for Occupational Safety and Health among employees of the Agricultural Research Service, were usually effective in either eliminating symptoms or at least leaving them amenable to pharmacological intervention. Recommendations addressing the prevention of sensitising exposures in insect raising facilities ${ }^{162324}$ are supported by the findings of our study and should include an adequate air extraction system, the use of laminar flow cabinets, implementation of an overall cleaning plan with a workspace designed for ease of washing down, a building plan designed to avoid exposing other workers, including office staff, and the provision of protective equipment including gloves, gowns, masks, eye protection, and respirators where necessary. Failure of these measures to control symptoms associated with persistence or worsening of serious symptoms (such as asthma), despite appropriate treatment, may sometimes necessitate a change of work.

In uncontrolled studies with fly larval extracts, desensitisation by subcutaneous injection has been reported to give favourable results. ${ }^{610}$ This remains unproved and invites further study.

We thank Gail Knowland for technical help. This study was funded by grants from the Commonwealth Scientific and Industrial Research Organization and the National Health and Medical Research Council of Australia.

\section{References}

1 Jamieson HC. The house fly as a cause of nasal allergy. $J$ Allergy 1938;9:273-4.

2 Tee RD, Gordon DJ, Lacey J, Nunn AJ, Brown M, Newman Taylor AJ. Occupational allergy to the common house fly (Musca domestica): use of immunological response to identify atmospheric allergen. J Allergy Clin Immunol 1985;76:826-31.

3 Weil CK. Hay-fever due to the scales of the tanytarsus. International Correspondence Society of Allergy 1938:63.

4 Kern RA. Asthma due to sensitization to a mushroom fly (Aphiochaeta agarici). J Allergy 1938;9:604-6.

5 Ordman D. Sewage filter flies (Psychoda) as a cause of bronchial asthma. S Afr Med J 1946;20:32-5.

6 Mariani G, Mariani M. L'iposensibilizzazione nelle allergie da larve di mosca (carnaria). Folia Allergol 1970;17:179-80.

7 Trinca JC. Insect allergy in Australia: results of a fiveyear survey. Med J Aust 1964;2:659-63.

8 Douglas RB. The hazards of pooting insects. Antenna 1984;8:193-4.

9 Bellas TE. Inhalant allergy to insects. In: Baldo BA, Howden MEH, eds. Proceedings of the Sydney Allergen Group. Vol 3. North Ryde: Macquarie University, 1983:48-55.

10 Gibbon HL, Dille JR, Cowley RG. Inhalant allergy to the screwworm fly, preliminary report. Arch Environ Health 1965;10:424-30.

11 Herrmann GHC. Allergy to screwworm fly dust. A new occupational disease. Texas State J Med 1966;62:36-9.

12 Dille JR, Gibbons HL, Spikes GA. Allergic problems in screwworm fly eradication program personnel. Aerospace Med 1968;39:1116-9.

13 Kaufman GL, Baldo BA, Tovey ER, Bellas TE, Gandevia BH. Inhalant allergy following occupational exposure to blowflies. Clin Allergy 1986;16:65-71.

14 Walsh BJ, Sutton R, Wrigley CW, Baldo BA. Allergen discs prepared from nitrocellulose: detection of IgE binding to soluble and insoluble allergens. Journal of Immunological Methods 1984;73:139-45.

15 Burge PS, Edge G, O'Brien IM, Harries MG, Hawkins R, 
Pepys $\mathrm{J}$. Occupational asthma in a research centre breeding locusts. Clin Allergy 1980;10:355-63.

16 Bauer M, Patnode R. National Institute of Occupational Safety and Health. Health hazard evaluation report HETA-81-121-1421, 1984. (Reproduced by National Technical Information Service, US Department of Commerce.)

17 Spieksma FThM, Vooren PH, Kramps JA, Dijkman JH. Respiratory allergy to laboratory fruit flies (Drosophila melanogaster). J Allergy Clin Immunol 1986;77: 108-13.

18 Edge G, Burge PS. Immunological aspects of allergy to locusts and other insects. Clin Allergy 1980;10:347 (abstract).

19 Baldo BA, Panzani RC. Detection of IgE antibodies to a wide range of insect species in subjects with suspected inhalant allergies to insects. Int Arch Allergy Appl Immunol 1988;85:278-87.

20 Buisseret P. Seasonal asthma in an angler. Lancet 1978;i:668.

21 Bellas TE. Inhalant allergy to insects-occupational aspects. In: Baldo BA, Harle DG, eds. Proceedings of the Sydney Allergen Group. Vol 5. Sydney: University

22 Frankland AW, Lessof MH. Allergy, insects and arachnids. In: Immunological and clinical aspects of allergy. Lancaster: MTP Press, 1981:373-88.

23 Wirtz RA. Occupational allergies to arthropods- $\vec{\circ}$ documentation and prevention. Bulletin of the $\overrightarrow{\vec{C}}$ Entomological Society of America 1980;26:356-60.

24 Work-related allergies in insect-raising facilities. $M M W R$ $1984 ; 33: 448,453-4$. 